DOI 10. 18307/2018. 0509

(C) 2018 by Journal of Lake Sciences

\title{
“堑秋湖”围堤改变鄱阳湖洲滩湿地土壤碳循环过程”
}

\author{
沈瑞昌 ${ }^{1,2,3,4}$, 兰志春 ${ }^{1,4,5}$, 方长明 ${ }^{2}$, 陈家宽 ${ }^{2 * *}$ \\ (1: 南昌大学生命科学研究院流域生态学研究所,南昌 330031) \\ (2:复旦大学生物多样性科学研究所, 上海 200433) \\ (3: 南昌大学鄱阳湖环境与资源利用教育部重点实验室,南昌 330031) \\ (4:江西鄱阳湖湿地生态系统国家定位观测研究站,南昌 330038) \\ ( 5 : 流域生态与地理环境监测国家测绘地理信息局重点实验室,南昌 330209)
}

\begin{abstract}
摘 要: 水文节律是影响湖泊湿地土壤碳循环的重要因素. “堑秋湖” 是鄱阳湖传统的渔业生产方式, 对鄱阳湖碟形湖水 文节律具有显著影响. 因此,本文研究了“堑秋湖”对鄱阳湖洲滩土壤碳循环的影响. 结果表明, “堑秋湖”围堤内外洲滩 土壤总有机碳含量无显著差异, 但围堤内土壤轻组有机碳含量比围堤外低 $35.03 \%$. 围堤内土壤有机碳 (SOC) 矿化速率明 显高于围堤外. 围堤内 SOC 矿化的平均 $Q_{10}$ 为 2.72 , 比围堤外低 $4.83 \%$, 但两者 SOC 矿化的水分敏感性没有显著差异. 围 堤内外 SOC 矿化速率分别与土壤可溶性有机碳和土壤轻组氮含量相关关系最为显著. 综上所述, “堑秋湖” 围堤已经改变 了鄱阳湖洲滩的土壤碳循环过程. 模拟湖泊湿地土壤碳循环时需要充分考虑当地渔业生产的影响.
\end{abstract}

关键词: 湿地; 土壤碳循环; 水文节律;渔业生产;气候变化;鄱阳湖

\section{The dikes of the sub-lakes change soil carbon cycle in the littoral wetland in Poyang Lake}

\author{
SHEN Ruichang ${ }^{1,2,3,4}$, LAN Zhichun ${ }^{1,4,5}$, FANG Changming ${ }^{2} \&$ CHEN Jiakuan ${ }^{2 * *}$ \\ (1: Center for Watershed Ecology, Institute of Life Science, Nanchang University, Nanchang 330031, P.R.China) \\ (2: Institute of Biodiversity Science, Fudan University, Shanghai 200433, P.R.China) \\ (3: Key Laboratory of Poyang Lake Environment and Resource Utilization, Ministry of Education, Nanchang University, Nan- \\ chang 330031, P.R. China) \\ (4: National Ecosystem Research Station of Jiangxi Poyang Lake Wetland, Nanchang 330038, P.R. China) \\ (5: Key Laboratory of Watershed Ecology and Geographical Environment Monitoring, NASG, Nanchang 330209, P.R.China)
}

Abstract: Water regime changes caused by natural and anthropogenic activities can significantly affect soil carbon cycle in wetland ecosystem. Some fishery activities could greatly impact on wetland water regime, but, till now, their influences on wetland soil carbon cycle are still not fully understood. In this paper, we investigated the effects of the dike of Lake Dahuchi, which was used to restrain the fish at the low water period, on soil carbon cycle in the littoral wetland. Our results showed that soil total organic carbon ( TOC) was not changed by the dike, but soil light fraction of organic carbon inside the dike was significantly lower than that outside the dike by $35.03 \%$. Meanwhile, the littoral wetland soil inside the dike had higher soil organic carbon (SOC) mineralization rate, but lower temperature sensitivity. The $Q_{10}$ of SOC mineralization inside and outside the dike were 2.72 and 2.85 , respectively. The dike did not significantly change the moisture sensitivity of SOC mineralization. In addition, the determining factors of SOC mineralization rates inside and outside the dike were dissolved organic carbon and light fraction of total nitrogen, respectively. In summary, soil carbon cycle in littoral wetland in Poyang Lake could be modified by fishery activities. Scientists should take account of the effects of fishery in simulating soil carbon cycle of the wetland ecosystem in future.

* 国家自然科学基金项目(31600381)、中国博士后科学基金面上项目(2017M620129)、江西省科技厅青年基金项目 (20171BAB214008) 和流域生态与地理环境监测国家测绘地理信息局重点实验室开放课题 (WE2015009) 联合资 助. 2017-11-28 收稿; 2018-01-22 收修改稿. 沈瑞昌 (1987 ), 男, 博士, 助理研究员; E-mail: ever412024629@ 163.com.

** 通信作者;E-mail:jkchen@fudan.edu.cn. 
Keywords: Wetland; soil carbon cycle; water regime; fishery; climate change; Poyang Lake

湿地是一个水陆相互作用形成的独特生态系统. 由于具有较高的生产力和较低的有机质分解速率, 湿 地土壤已经成为陆地生态系统中主要的碳库之一 ${ }^{[1]}$. 研究表明, 湿地生态系统虽然只占有陆地表面的 $4 \%$ $6 \%$, 却保有全球陆地生态系统土壤 $20 \% \sim 30 \%$ 的有机碳储量 ${ }^{[2-3]}$. 因此, 湿地土壤碳循环过程, 比如土壤总有 机碳含量、土壤有机碳各组分含量以及土壤有机碳矿化速率等的细微改变都可能引起大气 $\mathrm{CO}_{2}$ 浓度的巨大 变化, 从而对全球气候产生重要影响. 湿地生态系统土壤碳循环过程复杂而敏感, 受到许多气象和环境因子 的深刻影响. 水文节律就是其中一个重要的因子. 科学家已经在世界各地, 如亚极地 ${ }^{[4]}$ 、温带 ${ }^{[5]}$ 、热带 ${ }^{[6]}$ 等, 报道了水文节律对湿地土壤碳循环的影响. 在国内, 相关研究也在东北 ${ }^{[7]}$ 、华北 ${ }^{[8]}$ 、西北 ${ }^{[9]} 、$ 华南 ${ }^{[10]} 、$ 华 东 ${ }^{[11]}$ 、西南 ${ }^{[12]}$ 和青藏高原 ${ }^{[13]}$ 等地的湿地相继开展. 它们既有野外观测实验也有实验室培养实验, 主要关注 了自然水文节律对湿地土壤碳循环的影响, 比如土壤含水量、水淹时长 ${ }^{[9]}$ 、水淹频率 ${ }^{[10]}$ 、水位深度 ${ }^{[14]}$ 、干湿 交替 ${ }^{[15]}$ 和冻融作用 ${ }^{[16]}$ 等. 此外, 许多文献还报道了全球变化 (例如泥炭水位下降等 ${ }^{[17-18]}$ 和湿地管理 (例如 湿地围剭和恢复等 $)^{[19-20]}$ 如何通过改变水文节律影响湿地土壤碳循环过程. 然而, 在区域尺度上对水文节律 有重要影响的人为活动, 比如渔业活动, 如何影响湿地土壤碳循环过程还未受到重视. 鱼类资源是湿地生态 系统供给服务中重要的组成部分. 在湿地中开展渔业生产已经普遍存在. 部分渔业生产活动会引起湿地蓄 放水节律的显著变化. 它们可能会对局域土壤碳过程产生显著影响, 从而增加湿地生态系统土壤碳循环的 空间异质性,进而改变区域尺度湿地土壤碳循环过程.

鄱阳湖位于长江中下游平原, 是我国面积最大的淡水湖泊. “堑秋湖” 是鄱阳湖传统的渔业生产方式. 它 利用鄱阳湖湖盆内碟形湖泊众多的特点,通过修建或加固围堤 (即 “堑秋湖” 围堤) 的方式使枯水期本应流 走的水体保留在碟形湖中, 并在碟形湖水闸处捕获渔业资源 ${ }^{[21-22]}$. “堑秋湖” 围堤以内的洲滩湿地的水文节 律与大湖区明显不同, 最显著的特点就是具有更长的水淹时间 ${ }^{[22]}$. 前期许多学者的研究表明, 自然水分梯 度对鄱阳湖洲滩湿地土壤碳循环过程影响巨大. 在鄱阳湖洲滩不同的高程区, 土壤有机碳含量、组分和矿化 速率明显不同 ${ }^{[23-24]}$. 然而, 与鄱阳湖洲滩湿地自然长期形成的水分梯度不同的是, “堑秋湖” 围堤引起的水分 节律差异往往只存在了较短时间. 目前, 尚不清楚 “堑秋湖” 围堤是否已经对鄱阳湖洲滩湿地土壤碳循环过 程产生了影响. 因此, 本研究的目的是分析 “堑秋湖” 围堤对鄱阳湖洲滩湿地土壤有机碳含量、组分及其矿化 等土壤碳循环的影响. 本研究结果将有助于了解鄱阳湖乃至长江中下游湖泊群洲滩湿地的碳循环过程, 为 制定区域尺度的温室气体排放政策提供依据.

\section{1 材料与方法}

\section{1 研究区域}

选择大湖池代表 “堑秋湖” 围堤内的湖泊, 它位于江西省鄱阳湖国家级自然保护区内, 面积为 $20.82 \mathrm{~km}^{2}$ (图 1). 枯水期间, 永吴公路将大湖池与主湖切断, 从而使大湖池形成一个完整的 “堑秋湖”湖泊. 永吴公路 大湖池段始修于 1992 年, 最初只有一条简易公路. 它于 2013 年得到升级, 形成了一条高达 $3 \mathrm{~m}$ 的围堤. 至样 品采集的 2017 年 4 月,永吴公路大湖池段已经修建了 25 年时间. 整个围堤虽留有 2 个孔洞, 但两侧水体仍 难以进行自由交换. 围堤两侧的水文特征存在着明显差别 (图 2). 每年 8 月开始围堤外水位随鄱阳湖主湖 水位 (图 2 中以星子站为代表) 而波动下降, 而大湖池由于围堤的存在能够保持较高的水位. 11 月后, 由于 候鸟保护和捕鱼的需要, 大湖池闸口开始大量放水, 水位也随之下降. 12 月后, 大湖池水位一直保持较低状 态, 但仍旧比主湖高. 到第 2 年 4 月左右, 主湖水位上升, 大湖池与主湖水位相接后, 水位也逐渐升高. 直到 8 月底, 大湖池水位一直与主湖水位持平. 因此, 大湖池洲滩湿地每年的水淹时长要大于围堤外洲滩. 按照 1985 年国家高程基准, 研究样地的平均高程为 13.19 m. 2016 年, 大湖池洲滩湿地的平均淹沉时间为 $221 \mathrm{~d}$, 而围堤外洲滩湿地的平均淹沉时间为 $141 \mathrm{~d}$.

\section{2 样方设置与土样采集}

采样样方设置于永吴公路大湖池段两侧 (图 1). 在公路两侧, 各均匀布置 5 条 $100 \mathrm{~m}$ 的样带. 每条样带 均垂直于公路, 相邻样带间隔约 $0.5 \mathrm{~km}$. 由于公路两侧前均挖有宽约 $3 \mathrm{~m}$ 的水沟, 因此公路两侧样带的起点 


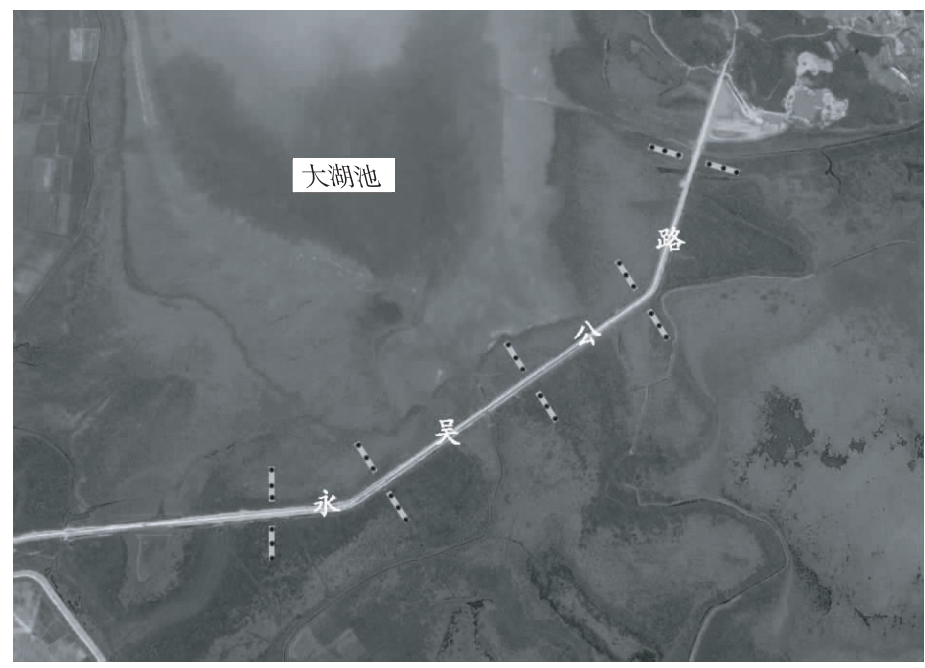

图 1 研究样带及样点布设 (灰色条带为样带, 其中的黑点为采样点)

Fig. 1 Location of study area and sampling sites (the gray strips mean line transects, the dark points mean sampling points)

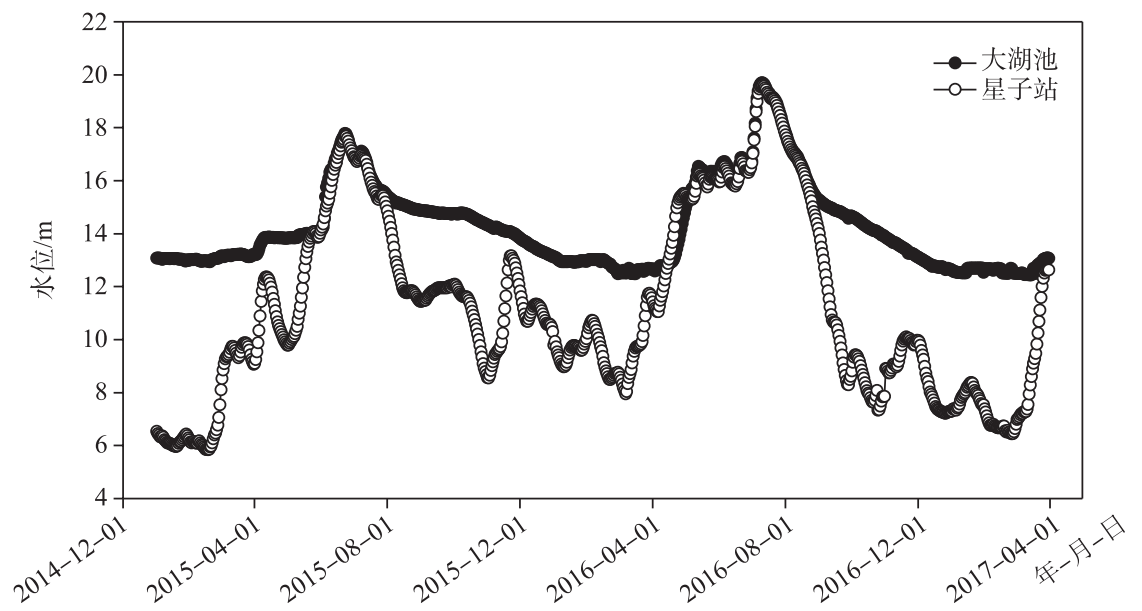

图 2 大湖池与星子站水位差异 (1985 年国家高程基准)

Fig.2 The water levels in Lake Dahuchi and Xingzi Hydrological Station (National Vertical Datum 1985)

均设置在距离水沟 $5 \mathrm{~m}$ 的地方. 在每条样带的第 $5 、 50 、 100 \mathrm{~m}$ 的位置上分别建立 1 个 $1 \mathrm{~m} \times 1 \mathrm{~m}$ 的样方. 利用 GPS 记录小样方的经纬度坐标. 利用瑞士徕卡 Sprinter $250 \mathrm{M}$ 电子水准仪在野外采点, 由江西省测绘局解译 样点的高程信息. 总体来讲, 本次实验共建立 $2 \times 5 \times 3=30$ 个小样方. 在同一条样带上, 围堤内外最远的样方 直线距离仅为 $300 \mathrm{~m}$ 左右, 保证了两者土壤、高程等条件的一致性. 此外, 由于公路两侧水沟的存在, 各样方 的可达性不高, 减少了它们受人为干扰的可能性. 由于近 25 年的水文条件的差异, 围堤内外样方的植物组 成和生物量产生了明显的差异 (图 3 ). 围堤内样方的植物以灰化苔草 (Carex cinerascens Kükenth.) 和融草 (Phalaris arundinacea Linn) 为主, 而围堤外样方则以南荻( Triarrhena lutarioriparia L Liu) 和灰化苔草为主.

本研究于 2017 年 4 月在每个小样方中采集土壤样品. 在每个样方的一条对角线上取 2 钻, 取样深度为 $0 \sim 10 \mathrm{~cm}$, 取样钻直径为 $7 \mathrm{~cm}$. 样品当天就被送回实验室. 在实验室中, 每个样方的土壤样品充分混合成一 个均匀的土壤样品, 通过 $2 \mathrm{~mm}$ 笁以过滤石块和根系. 


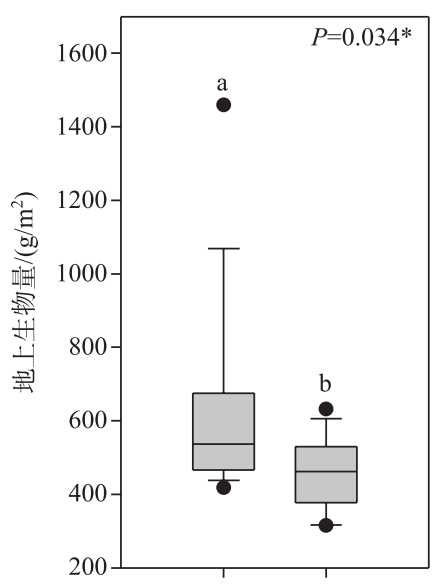

围堤内 围堤外

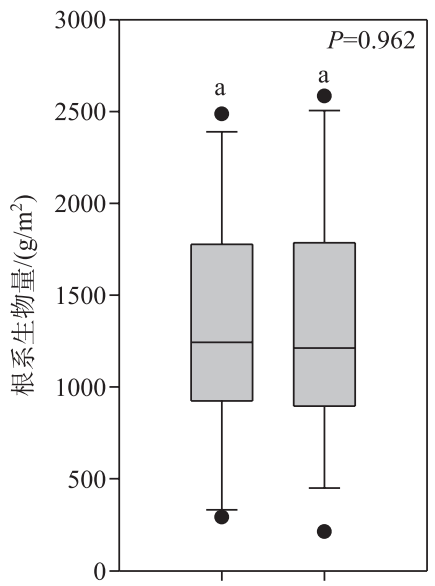

围堤内 围堤外

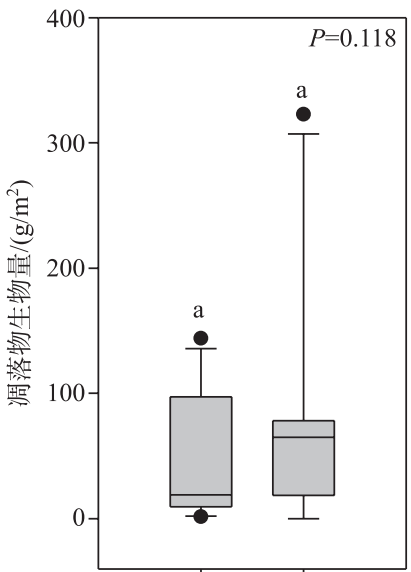

围堤内 围堤外

图 3 围堤内外样方中地上生物量、根系生物量及调落生物量的差异

Fig. 3 The differences of aboveground plant biomass, root biomass and litter biomass between the sampling sites inside and outside of the dike

\section{3 土壤理化参数的测定}

称取 $20 \mathrm{~g}$ 左右的土壤在 $105^{\circ} \mathrm{C}$ 下烘 $24 \mathrm{~h}$ 以测定土壤含水量. 土壤饱和持水量 ( WHC) 的测定方法改自 Rey 等 ${ }^{[25]}$, 具体是称取约 $30 \mathrm{~g}$ 新鲜土壤至环刀中, 在蒸馏水中浸泡 $24 \mathrm{~h}$, 再静滞 $24 \mathrm{~h}$ 使自由水充分流走, 最 后利用烘干法测定含水量, 此土壤含水量即为土壤饱和持水量. 按照土水比为 $1: 2.5(\mathrm{~W} / \mathrm{V})$ 的比例用 $\mathrm{pH}$ 计 (SevnMulti S40, Mettler-toledo Group, Switzerland) 测定土壤 $\mathrm{pH}$ 值. 土壤总有机碳( TOC) 和总氮( TN) 运用元 素分析仪 (Vario EL III, Elemenar, Germany) 测定. 由于南方酸性土壤特征, 本地区土壤中无机碳含量很低, 因此本研究没有考虑土壤无机碳对土壤有机碳结果的影响. 利用土壤 TOC 与 TN 的比值获得土壤碳氮比 $(\mathrm{C} / \mathrm{N})$. 土壤重组有机质与轻组有机质是按照 Janzen 等 ${ }^{[26]}$ 的方法以密度为 $1.7 \mathrm{~g} / \mathrm{cm}^{3}$ 的 NaI 溶液进行区分. 土壤重组有机碳( HFOC) 及重组总氮( HFN) 含量运用元素分析仪 (Vario EL III, Elemenar, Germany) 测定. 由于土壤中轻组有机质含量较低, 因此, 本研究运用土壤 TOC 与 HFOC 的差值计算土壤轻组有机碳 ( LFOC), 运用土壤 TN 与 HFN 的差值获得轻组氮 ( LFN $)^{[24]}$. 土壤可溶性有机碳 (DOC) 和可溶性总氮 (DTN) 则按照土水比 $1: 5(\mathrm{~W} / \mathrm{V})$ 的比例, 利用蒸馏水提取, 最后通过总有机碳分析仪 (Vario TOC, Elementar, Germany) 测定. 土壤重组有机碳比例 $\left(\mathrm{P}_{\mathrm{HFOC}}\right)$ 、轻组有机碳比例 $\left(\mathrm{P}_{\mathrm{LFOC}}\right)$ 和可溶性有机碳比例 $\left(\mathrm{P}_{\mathrm{DOC}}\right)$ 分别表征 HFOC、LFOC 和 DOC 占土壤 TOC 的比例. 土壤重组氮比例 $\left(\mathrm{P}_{\mathrm{HFN}}\right)$ 、轻组氮比例 $\left(\mathrm{P}_{\mathrm{LFN}}\right)$ 和可 溶性总氮比例 $\left(\mathrm{P}_{\mathrm{DTN}}\right)$ 分别表征 HFN 、LFN 和 DTN 占土壤 TN 的比例.

\section{4 土壤有机碳矿化速率的测定}

土壤有机碳矿化速率实验包括测定土壤有机碳矿化的温度敏感性和水分敏感性两部分. 温度敏感性实 验是将土壤培养于不同温度梯度下完成的. 称取相当于干土重 $50 \mathrm{~g}$ 的新鲜土壤于 $125 \mathrm{ml}$ 的玻璃瓶中, 调节 水分使土壤样品含水量达到 $65 \%$ 的饱和含水量 $(65 \% \mathrm{WHC}$ ). 将培养瓶在室温下静滞 $48 \mathrm{~h}$, 以消除由于土壤 扰动造成的分解加速现象对实验结果的影响. 运用一套土壤培养与 $\mathrm{CO}_{2}$ 通量测定系统测定土壤样品在不同 温度下的有机碳矿化速率. 该系统采用低温冷却槽对土壤样品进行控温, 土壤样品的实际温度由放置在另 一个样品瓶中的钮扣温度计 (DS1922L, maxim, USA) 进行测定. 数据显示, 当土壤培养系统的温度调整后, 土壤样品的温度能够在 $1.5 \mathrm{~h}$ 内达到新的平衡. 本次实验的温度梯度为 $5 、 10 、 15 、 20 、 25$ 和 $30^{\circ} \mathrm{C}$. 在每个温度 下, 运用红外分析仪 ( Li-820, Licor, USA) 对土壤样品进行 $\mathrm{CO}_{2}$ 通量的测定. 每个样品在每个温度下用红外 分析仪记录 $3 \mathrm{~min} \mathrm{CO}_{2}$ 浓度数据, 其中采用后 $2 \mathrm{~min}$ 数据分析土壤样品的 $\mathrm{CO}_{2}$ 通量. 本文将用 $\mathrm{R}_{5} 、 \mathrm{R}_{10} 、 \mathrm{R}_{15}$ 、 $R_{20} 、 R_{25} 、 R_{30}$ 分别指代 $5 、 10 、 15 、 20 、 25$ 和 $30^{\circ} \mathrm{C}$ 培养下的土壤有机碳矿化速率. 运用指数方程拟合土壤有机碳 
矿化的温度敏感性 $\left(Q_{10}\right)$.

土壤有机碳矿化的水分敏感性是指土壤有机碳矿化速率对土壤水分变化的敏感程度. Hawkes 等 ${ }^{[27]}$ 将 土壤有机碳矿化速率与水分线性拟合方程的斜率看作土壤有机碳矿化的水分敏感性. 他们发现不同地区土 壤有机碳矿化的水分敏感性并不相同,长期湿润区域比长期干燥区域土壤有机矿碳化的水分敏感性要高. 因此,本研究同样分析了“堑秋湖” 围堤引起的水文节律变化是否已经改变土壤有机碳矿化的水分敏感性. 采用 Hawkes 等 ${ }^{[27]}$ 的研究方法测定土壤有机碳矿化的水分敏感性. 土壤样品先进行风干使其含水量降至 $20 \%$ WHC 以下. 称取 5 份相当于 $40 \mathrm{~g}$ 干重的土壤样品放人 5 个 $125 \mathrm{ml}$ 的样品瓶, 然后将其水分分别调至 $20 \%$ WHC、 $45 \%$ WHC、 $70 \%$ WHC、 $95 \%$ WHC 和 $120 \%$ WHC 5 个水平. 调水后的样品瓶放置在室温下培养, 定期 根据重量的差异补充水分. 在调水后的第 $9 、 14$ 和 24 天, 将样品瓶放置在土壤培养与 $\mathrm{CO}_{2}$ 通量测定系统中, 测定土壤有机碳的矿化速率. 土样培养温度设定为 $20^{\circ} \mathrm{C}$. 由于各土壤样品有机碳矿化的最适水分不一致, 可 能是 $70 \% \mathrm{WHC} 、 95 \% \mathrm{WHC}$ 或 $120 \% \mathrm{WHC}$. 因此, 仅将土壤有机碳矿化速率与 $20 \% \mathrm{WHC} 、 45 \% \mathrm{WHC}$ 和 70\% WHC 3 个水分梯度线性关系的斜率当作土壤有机碳矿化的水分敏感性. 3 次测定得到的水分敏感性分别记 录为 $k_{1} 、 k_{2}$ 和 $k_{3}$.

\section{5 数据分析}

采用单因素方差分析法 (One-way ANOVA) 判定 “堑秋湖” 围堤对土壤有机碳含量、组分和矿化相关参数 的影响. 利用相关关系分析高程与围堤内外土壤有机碳含量、组分和矿化相关参数的相关关系. 采用逐步回 归分析方法分析 $\mathrm{pH} 、 T O C 、 T N 、 C / N 、 H F O C 、 H F N 、 L F O C 、 L F N 、 D O C 、 D T N 、 P_{H F O C 、} P_{L F O C} 、 P_{D O C} 、 P_{L F N} 、 P_{H F N}$ 和 $P_{D T N}$ 等土壤理化参数对围堤内外土壤有机碳矿化速率及其温度敏感性和水分敏感性的方差的解释能力. 由于围 堤内 $\mathrm{pH} 、 T O C 、 T N 、 H F O C 、 H F N 、 D O C 、 D T N$ 以及围堤外的 LFN 等数据不符合正态分布, 所以在回归分析前对 这些数据进行对数转换以符合正态分布规律. 所有统计分析都在 SPSS 22( IBM, USA) 软件中完成.

\section{2 结果与分析}

\section{1 土壤有机碳及其组分}

“堑秋湖” 围堤内外土壤有机碳含量差异很小, 但围堤内土壤有机碳含量的空间异质性明显较高. “堑秋 湖” 围堤对土壤 TOC 没有显著影响 $(P=0.786)$. 围堤外土壤 TOC 的变化范围为 $1.32 \% \sim 3.14 \%$, 平均值为 $2.32 \%$, 变异系数 $(C V)$ 仅为 $22.33 \%$. 而围堤内 TOC 变化范围为 $0.88 \% \sim 6.82 \%$, 平均值为 $2.43 \%, C V$ 达到 $61.58 \%$. 同时, “堑秋湖” 围堤没有显著改变土壤 HFOC、DOC、 $\mathrm{P}_{\mathrm{HFOC}} 、 \mathrm{P}_{\mathrm{LFOC}}$ 和 $\mathrm{P}_{\mathrm{DOC}}$, 但是围堤内土壤的 LFOC 显著低于围堤外土壤 $(P=0.006)$. 围堤外表层土壤 HFOC、LFOC 以及 DOC 平均值分别为 $1.92 \% 、 0.40 \%$ 和 $36.14 \mu \mathrm{g} \mathrm{C} / \mathrm{g}$ dry soil, 分别占总有机碳的 $82.31 \% 、 17.69 \%$ 和 $0.16 \%, C V$ 值分别为 $24.88 \% 、 32.18 \%$ 和 $33.22 \%$. 围堤内表层土壤 HFOC、LFOC 以及 DOC 平均值分别为 $2.17 \% 、 0.26 \%$ 和 $35.18 \mu \mathrm{g} \mathrm{C} / \mathrm{g}$ dry soil, 分别占总有机 碳的 $87.01 \% 、 12.99 \%$ 和 $0.16 \%, C V$ 值分别为 $66.18 \% 、 50.28 \%$ 和 $46.75 \%$ (表 1 ).

表 1 “堑秋湖”围堤内外表层土壤有机碳及其组分含量

Tab.1 The amounts of soil organic carbon and its components beside the dike of a sub-lake

\begin{tabular}{|c|c|c|c|c|c|c|}
\hline \multirow{2}{*}{ 参数 } & \multicolumn{2}{|c|}{ 平均值 } & \multicolumn{2}{|c|}{ 标准差 } & \multicolumn{2}{|c|}{ 变异系数 } \\
\hline & 围堤内 & 围堤外 & 围堤内 & 围堤外 & 围堤内 & 围堤外 \\
\hline TOC/\% & $2.43^{\mathrm{a}}$ & $2.32^{\mathrm{a}}$ & 1.50 & 0.52 & $61.58 \%$ & $22.33 \%$ \\
\hline HFOC/\% & $2.17^{\mathrm{a}}$ & $1.92^{\mathrm{a}}$ & 1.43 & 0.48 & $66.18 \%$ & $24.88 \%$ \\
\hline LFOC/\% & $0.26^{\mathrm{a}}$ & $0.40^{\mathrm{b}}$ & 0.13 & 0.13 & $50.28 \%$ & $32.18 \%$ \\
\hline $\mathrm{DOC} /(\mu \mathrm{g} \mathrm{C} / \mathrm{g}$ dry soil $)$ & $35.18^{\mathrm{a}}$ & $36.14^{\mathrm{a}}$ & 16.45 & 12.00 & $46.75 \%$ & $33.22 \%$ \\
\hline $\mathrm{P}_{\mathrm{HFOC}} / \%$ & $87.01^{\mathrm{a}}$ & $82.31^{\mathrm{a}}$ & 9.92 & 5.01 & $11.41 \%$ & $6.08 \%$ \\
\hline $\mathrm{P}_{\mathrm{LFOC}} / \%$ & $12.99^{\mathrm{a}}$ & $17.69^{\mathrm{a}}$ & 9.92 & 5.01 & $76.37 \%$ & $28.29 \%$ \\
\hline $\mathrm{P}_{\mathrm{DOC}} / \%$ & $0.16^{\mathrm{a}}$ & $0.16^{\mathrm{a}}$ & 0.05 & 0.03 & $32.20 \%$ & $20.66 \%$ \\
\hline
\end{tabular}

* 平均值一列中同一个字母表示围堤内外没有显著差异 $(P>0.05)$, 不同的字母显示围堤内外有显著差异 $(P<0.05)$. 


\section{2 土壤有机碳矿化}

与围堤外相比, 围堤内土壤有机碳矿化的速率明显偏高, 但温度敏感性则偏低. 土壤有机碳矿化速率随 温度的升高而呈指数增长的趋势 (图 4A). 围堤内外土壤有机碳矿化速率都呈现一定的空间异质性. 围堤外 $\mathrm{R}_{5} \sim \mathrm{R}_{30}$ 的 $C V$ 值介于 $31.75 \% \sim 46.46 \%$ 之间，围堤内 $\mathrm{R}_{5} \sim \mathrm{R}_{30}$ 的 $C V$ 值则相对较高, 介于 $46.08 \% \sim 55.65 \%$ 之间. 在各个培养温度下, 围堤内土壤有机碳矿化速率都有高于围堤外的趋势. 但是只有 $\mathrm{R}_{10}$ 在围堤内外的差异显 著 $(P=0.047)$, 围堤内的 $\mathrm{R}_{10}$ 比围堤外 $\mathrm{R}_{10}$ 高出了 $45.47 \%$. 不过, 围堤内土壤有机碳矿化的温度敏感性显著 低于围堤外 $(P=0.040), Q_{10}$ 的平均值分别为 2.72 和 2.85 (图 4B).

然而, 围堤内外土壤有机碳矿化的水分敏感性并没有显著差异 (图 5). 总体上,土壤有机碳矿化速率随 着土壤水分的增加逐渐升高. 但对于单个土壤样品, 土壤有机碳矿化速率随土壤水分升高的变化趋势差异 显著. 土壤有机碳矿化速率的最适水分含量可能是 70\% WHC、95\% WHC 或 120\% WHC. 随着培养时间的增 加, 土壤有机碳矿化速率在逐渐降低, 水分敏感性也在相应下降. 在各个测定时期, 围堤内土壤有机碳矿化 速率的平均值都高于围堤外, 但差异都不显著 $(P>0.05)$. 与此同时, 围堤内外土壤有机碳矿化的水分敏感性 的差异在统计学上也不显著 $(P>0.05)$. 围堤内外 $\mathrm{k}_{1} 、 \mathrm{k}_{2}$ 和 $\mathrm{k}_{3}$ 的平均值分别为 $0.46 、 0.38$ 和 0.31 .
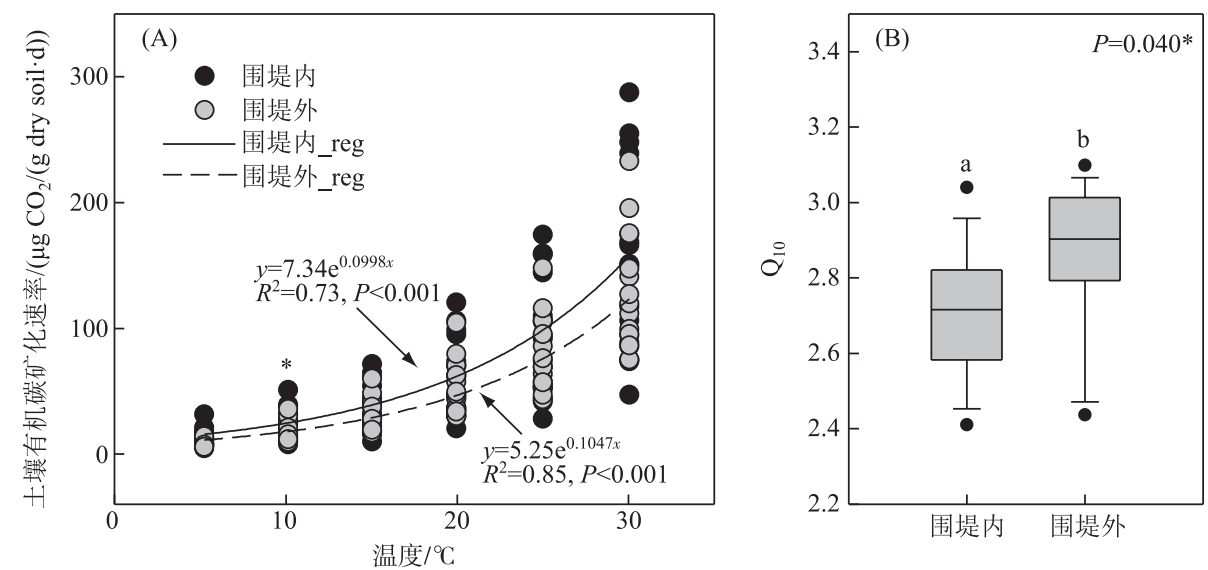

图 4 土壤有机碳矿化速率与温度间的关系以及围堤内外有机碳矿的温度敏感性

Fig.4 Relationships between soil organic carbon mineralization rate and temperature and the temperature sensitivity of soil organic carbon mineralization

\section{3 土壤有机碳及其矿化与高程的关系}

围堤内外土壤有机碳含量及其组分与高程的关系 明显不同(表 2). 基于 1985 年国家高程基准,围堤内样 点的高程范围为 $12.55 \sim 13.64 \mathrm{~m}$, 围堤外样点的高程范 围为 $12.82 \sim 13.86 \mathrm{~m}$. 围堤外土壤 TOC 和 HFOC 与高程 呈反比例关系, 相关系数分别为 -0.57 和 -0.60 ( TOC: $P=$ 0.025 ; HFOC: $P=0.017)$. 其他土壤有机碳组分参数与高 程的相关关系则不显著. 而围堤内土壤有机碳及其组分 参数中, 只有 $\mathrm{LFOC}$ 与高程的相关关系在统计上是显著 的, 相关系数为 $0.59(P=0.019)$. 与此同时, 无论是围堤 外还是围堤内, 土壤有机碳矿化的速率 $\left(\mathrm{R}_{5} 、 \mathrm{R}_{10} 、 \mathrm{R}_{15}\right.$ 、 $\left.R_{20} 、 R_{25} 、 R_{30}\right) 、 Q_{10}$ 和水分敏感性 $\left(k_{1} 、 k_{2}\right.$ 和 $\left.k_{3}\right)$ 与高程的 相关关系都不高. 只有围堤外 $\mathrm{R}_{5}$ 以及 $\mathrm{k}_{1}$ 和 $\mathrm{k}_{2}$ 与高程呈 反比例关系, 相关系数分别为 $-0.57(P=0.026) 、-0.51$ $(P=0.050)$ 和 $-0.57(P=0.028)$.
表 2 高程与土壤有机碳含量、 组分及矿化过程的相关关系

Tab.2 Relationships between elevation and some soil organic carbon related variables

\begin{tabular}{ccc||ccc}
\hline 位置 & 围堤内 & 围堤外 & 位置 & 围堤内 & 围堤外 \\
\cline { 3 - 5 } TOC & 0.29 & $-0.57^{*}$ & $\mathrm{R}_{15}$ & 0.14 & -0.48 \\
HFOC & 0.25 & $-0.60^{*}$ & $\mathrm{R}_{20}$ & 0.14 & -0.43 \\
LFOC & $0.59^{*}$ & -0.10 & $\mathrm{R}_{25}$ & 0.15 & -0.39 \\
DOC & 0.26 & -0.37 & $\mathrm{R}_{30}$ & 0.15 & -0.42 \\
$\mathrm{P}_{\text {HFOC }}$ & -0.38 & -0.34 & $\mathrm{Q}_{10}$ & -0.05 & 0.34 \\
$\mathrm{P}_{\text {LFOC }}$ & 0.38 & 0.34 & $\mathrm{k}_{1}$ & 0.07 & $-0.51^{*}$ \\
$\mathrm{P}_{\mathrm{DOC}}$ & -0.09 & 0.06 & $\mathrm{k}_{2}$ & 0.03 & $-0.57^{*}$ \\
$\mathrm{R}_{5}$ & 0.20 & $-0.57^{*}$ & $\mathrm{k}_{3}$ & -0.08 & -0.34 \\
$\mathrm{R}_{10}$ & 0.12 & -0.47 & & & \\
\hline
\end{tabular}



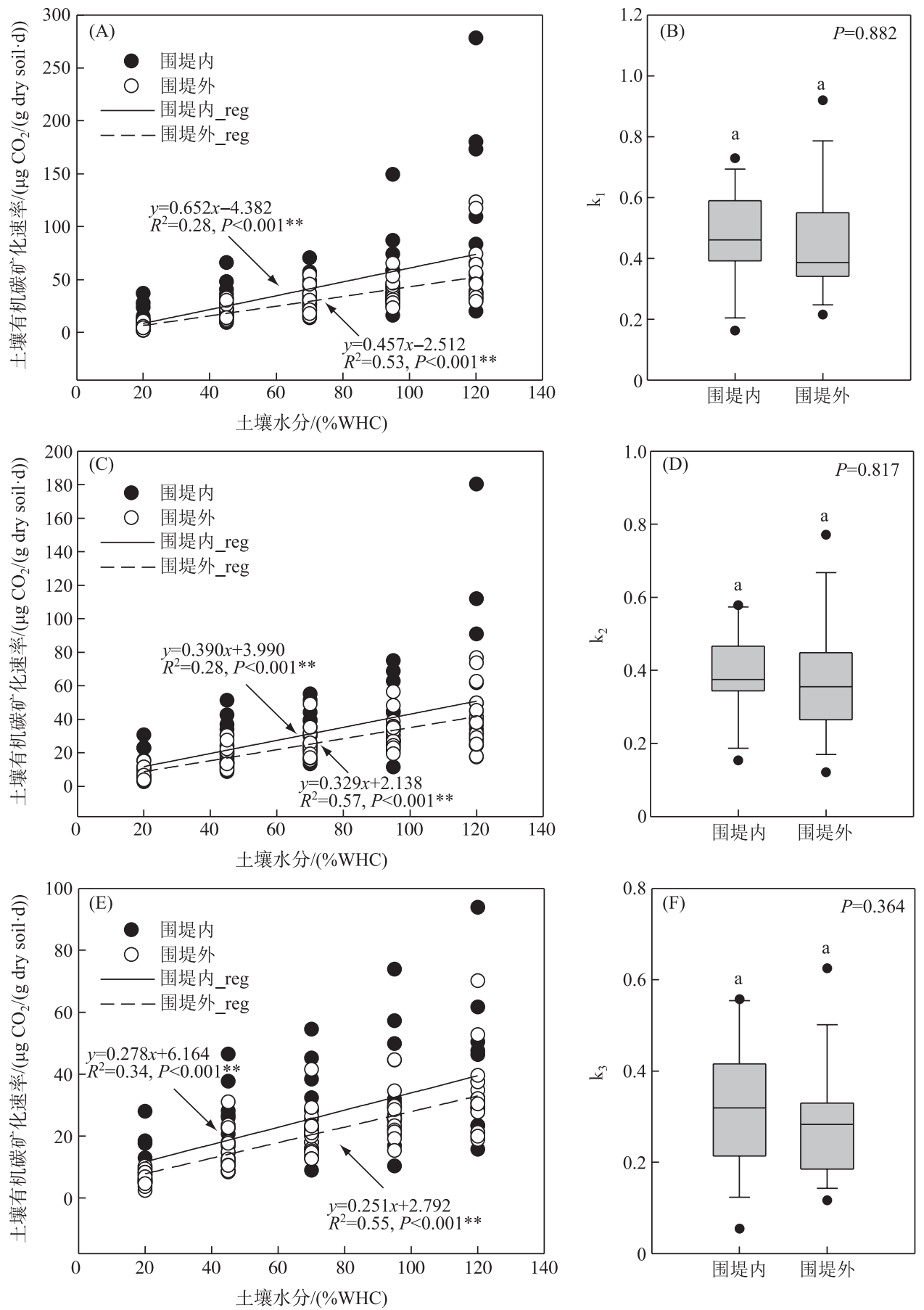

图 5 实验室培养条件下土壤有机碳矿化速率受土壤水分的影响

( $\mathrm{A}$ 和 $\mathrm{B}$ 是培养第 9 天后的结果; $\mathrm{C}$ 和 $\mathrm{D}$ 是培养第 14 天后的结果; $\mathrm{E}$ 和 $\mathrm{F}$ 是培养第 24 天后的结果)

Fig.5 Relationships between soil organic carbon (SOC) mineralization rate and soil moisture and moisture sensitivity of SOC mineralization in the incubation experiment (9th day: A and B; 14th day: C and D; 24th day: E and F) 


\section{4 土壤有机碳矿化的影响因素分析}

“堑秋湖”围堤显著改变鄱阳湖洲滩湿地土壤有机碳矿化速率的影响因子. 多元回归分析结果表明土壤 理化参数能够较好地解释土壤有机碳矿化速率的方差, 决定系数多数在 0.60 以上. 而且, 培养温度越高, 围 堤内外土壤有机碳矿化回归方程的决定系数有增加的趋势. 围堤内回归方程的决定系数普遍高于围堤外. 不过, 围堤内外多元回归方程的参数明显不同. 围堤外对土壤有机碳矿化速率的方差解释能力最强的参数 为 HFN 或 $\mathrm{P}_{\mathrm{HFN}}$; 而围堤内回归方程的参数则是 $\mathrm{DOC}$ (表 3). 在围堤内外, 土壤理化参数对土壤有机碳矿化 的温度敏感性的解释能力都非常有限, 在统计上都不显著. 而土壤有机有机碳矿化的水分敏感性主要与土 壤 DOC 含量有关. 随着土壤 DOC 含量的升高,水分敏感性越大(表 3 ).

表 3 土壤有机碳分解速率及水分敏感性的逐步多元回归分析结果

Tab.3 Results of the multivariate regression analyses on soil organic carbon mineralization rate related variables with soil physical and chemical variables

\begin{tabular}{|c|c|c|c|c|c|c|c|c|c|c|}
\hline \multirow{2}{*}{$\begin{array}{l}\text { 拟合 } \\
\text { 参数 }\end{array}$} & \multicolumn{5}{|c|}{ 围堤内 } & \multicolumn{5}{|c|}{ 围堤外 } \\
\hline & 自变量 & $\begin{array}{l}\text { 非标准 } \\
\text { 化系数 }\end{array}$ & $\begin{array}{l}\text { 标准 } \\
\text { 系数 }\end{array}$ & $R_{\text {adj }}^{2}$ & $P$ & 自变量 & $\begin{array}{l}\text { 非标准 } \\
\text { 化系数 }\end{array}$ & $\begin{array}{l}\text { 标准 } \\
\text { 系数 }\end{array}$ & $R_{\text {adj }}^{2}$ & $P$ \\
\hline \multirow[t]{2}{*}{$\mathrm{R}_{5}$} & 截距 & -29.775 & & 0.63 & $<0.001$ & 截距 & -13.817 & & 0.46 & 0.003 \\
\hline & $\lg ($ DOC $)$ & 28.198 & 0.810 & & & PLFN & -0.376 & 0.708 & & \\
\hline \multirow[t]{2}{*}{$\mathrm{R}_{10}$} & 截距 & -52.089 & & 0.70 & $<0.001$ & 截距 & -44.051 & & 0.62 & $<0.001$ \\
\hline & $\lg ($ DOC $)$ & 51.190 & 0.851 & & & $\lg (\mathrm{LFN})$ & -39.536 & -0.805 & & \\
\hline \multirow[t]{2}{*}{$\mathrm{R}_{15}$} & 截距 & -76.579 & & 0.67 & $<0.001$ & 截距 & -69.596 & & 0.61 & $<0.001$ \\
\hline & $\lg ($ DOC $)$ & 76.522 & 0.835 & & & $\lg (\mathrm{LFN})$ & -63.751 & -0.801 & & \\
\hline \multirow[t]{2}{*}{$\mathrm{R}_{20}$} & 截距 & -124.995 & & 0.70 & $<0.001$ & 截距 & -121.467 & & 0.65 & $<0.001$ \\
\hline & $\lg ($ DOC $)$ & 126.402 & 0.851 & & & $\lg (\mathrm{LFN})$ & -110.649 & -0.821 & & \\
\hline \multirow[t]{2}{*}{$\mathrm{R}_{25}$} & 截距 & -199.126 & & 0.74 & $<0.001$ & 截距 & -164.462 & & 0.65 & $<0.001$ \\
\hline & $\lg ($ DOC $)$ & 197.393 & 0.869 & & & $\lg (\mathrm{LFN})$ & -154.527 & -0.820 & & \\
\hline \multirow[t]{3}{*}{$\mathrm{R}_{30}$} & 截距 & -308.864 & & 0.71 & $<0.001$ & 截距 & -278.984 & & 0.72 & $<0.001$ \\
\hline & $\lg ($ DOC $)$ & 310.213 & 0.852 & & & $\lg (\mathrm{LFN})$ & -228.919 & -0.779 & & \\
\hline & & & & & & DOC & 1.343 & 0.339 & & \\
\hline \multirow[t]{2}{*}{$\mathrm{k}_{1}$} & 截距 & -0.500 & & 0.67 & $<0.001$ & 截距 & -0.161 & & 0.66 & $<0.001$ \\
\hline & $\lg ($ DOC $)$ & 0.645 & 0.834 & & & HFN & 3.043 & 0.825 & & \\
\hline \multirow[t]{2}{*}{$\mathrm{k}_{2}$} & 截距 & -0.326 & & 0.64 & $<0.001$ & 截距 & -0.025 & & 0.62 & $<0.001$ \\
\hline & $\lg ($ DOC $)$ & 0.472 & 0.813 & & & DOC & 0.011 & 0.805 & & \\
\hline \multirow[t]{3}{*}{$\mathrm{k}_{3}$} & 截距 & -0.352 & & 0.52 & 0.005 & 截距 & -0.015 & & 0.66 & $<0.001$ \\
\hline & $\lg ($ DOC $)$ & 0.538 & 0.745 & & & DOC & 0.008 & 0.827 & & \\
\hline & LFOC & -0.482 & -0.431 & & & & & & & \\
\hline
\end{tabular}

\section{3 讨论}

渔业生产活动在长江中下游湖泊群中广泛存在, 它们极大地改变着洲滩湿地的水文节律. 本研究结果 显示 “堑秋湖” 围堤通过影响水文节律已经显著改变了鄱阳湖洲滩湿地的土壤碳循环过程. 相对于围堤外洲 滩土壤, 围堤内洲滩土壤有机碳含量的变异系数明显偏高; 土壤有机矿化速率更高, 特别是 $\mathrm{R}_{10}$ 高出 $45.47 \%$; 而土壤轻组有机碳含量则降低了 $35.03 \%$; 土壤有机碳矿化温度敏感性也要小 $4.83 \%$. 与此同时, 土壤有机碳 矿化速率与土壤理化因子关系也明显不同. 围堤内土壤有机碳矿化主要与土壤 DOC 含量有关, 而围堤外土 壤有机碳矿化则与 LFN 的相关关系更大. 因此, 局域尺度上的人类渔业活动对长江中下游湿地生态系统的 土壤碳循环过程有非常巨大的影响. 人们在模拟长江中下游湿地土壤碳循环过程时必须充分考虑当地渔业 生产措施对水节文律的影响.

“堑秋湖” 围堤内土壤有机碳矿化速率较高, 而轻组有机碳含量和有机碳矿化温度敏感性较低的原因可 
能是较高的土壤水分含量使围堤内土壤微生物能够更好地获取土壤轻组碳. 许秀丽等 ${ }^{[28]}$ 的研究表明鄱阳 湖洲滩湿地的地下水位与湖泊水位高度一致,地下水位高则土壤的含水量高. 在洲滩出露的同一时期,围堤 内湖泊的水位往往比围堤外湖泊的水位高, 因此, 围堤内土壤的含水量可能比围堤外高. 土壤水分构成了土 壤微生物生活的液相环境. 土壤水分的增加将提升土壤有机碳向微生物扩散的能力, 即增加土壤有机碳的 有效性 ${ }^{[29]}$. 而土壤微生物主要的基质就是土壤轻组有机碳. 因此, 围堤内较高的土壤有机碳矿化速率很可 能是土壤微生物能够获取更多的土壤轻组碳造成的. 这一解释机制也完全符合土壤有机碳矿化温度敏感性 的碳质量假说. 碳质量假说认为土壤有机碳矿化的温度敏感性由土壤有机碳质量决定, 土壤有机碳质量越 高则温度敏感性越低 ${ }^{[30-31]}$. 由于围堤内土壤微生物能够利用更多高质量的轻组有机碳, 所以其土壤有机碳 矿化的温度敏感性就比围堤外要低. 与此同时, 围堤内外由于水文节律差异引起的植被差异也可能对土壤 有机碳及其矿化过程有影响. 经过 25 年的水分条件差异后, 围堤内外植物组成和生物量存在着明显的差异. 围堤内洲滩的主要植物为灰化苔草和曧草, 而南荻和灰化苔草才是围堤外洲滩的主要建群种. 围堤内洲滩 地上植被生物量比围堤外洲滩显著高 $34.43 \%$. 植被是洲滩土壤有机碳的最主要来源. 更多的植物生物量供 应使围堤内土壤有机碳的矿化速率更高. 而且, 围堤内地上植被生物量的变异系数 ( $41.82 \%)$ 明显高于围堤 外的变异系数 $(20.32 \%)$, 这可能也是围堤内土壤有机碳含量具有更高变异系数的原因. 不过, 由于围堤内 较高的土壤有机碳分解速率以及更长时间的水分冲刷, 围堤内洲滩的凋落物含量并没有围堤外洲滩高. 调 查表明, 虽然由于样方间较大的变异使围堤内外洲滩的调落物含量没有显著差异, 但从平均值来看, 围堤内 洲滩凋落物含量只有 $43.50 \mathrm{~g} / \mathrm{m}^{2}$, 而围堤外洲滩调落物含量则为 $93.47 \mathrm{~g} / \mathrm{m}^{2}$. 较低的调落物含量可能减少了 土壤轻组有机碳的来源, 从而造成围堤内洲滩轻组有机碳含量较低.

此外, 围堤内外土壤有机碳矿化水分敏感性没有发生显著变化. 造成这一结果的原因可能包括两点: 第 一, 可能是因为 “堑秋湖” 围堤引起水文节律差异的时间还较短. Hawkes 等 ${ }^{[27]}$ 的研究表明只有长期降雨的 差异才能够使土壤有机碳矿化水分敏感性发生变化, 而且水分敏感性的差异能够持续非常长的时间. 本研 究中 “堑秋湖” 围堤建立了 25 年. 在围堤修建之前, 两边样地土壤基本保持着相同的水文节律. 随着围堤存 在时间的增加, 两侧土壤有机碳矿化水分敏感性的差异才可能显现. 第二, 可能是因为围堤两侧土壤微生物 群落进行了交流. 围堤两侧样方距离非常近. 在丰水期间, 围堤两侧表层土壤微生物可能随着泥沙的再悬浮 和沉积作用进行交流, 从而减弱它们的活性产生水分敏感性差异的可能. 与本研究区域不同的是, Hawkes 等 ${ }^{[27]}$ 的研究区域为一个大尺度的连续气候渐变区, 土壤微生物群落随降水的梯度具有明显的差异.

致谢: 非常感谢南昌大学生命科学学院的本科生许暳祯、史佩平和潘秀娟在实验过程中的帮助.

\section{4 参考文献}

[ 1 ] Dargie GC, Lewis SL, Lawson IT et al. Age, extent and carbon storage of the central Congo Basin peatland complex. Nature, 2017, $\mathbf{5 4 2}(7639)$ : 86-90.

[ 2 ] Post WM, Emanuel WR, Zinke PJ et al. Soil carbon pools and world life zones. Nature, 1982, 298(5870) : 156-159.

[ 3 ] Mitra S, Wassmann R, Vlek PLG. An appraisal of global wetland area and its organic carbon stock. Current Science, $2005, \mathbf{8 8}(1): 25-35$.

[ 4 ] Berglund O, Berglund K. Influence of water table level and soil properties on emissions of greenhouse gases from cultivated peat soil. Soil Biology \& Biochemistry, 2011, 43(5) : 923-931.

[ 5 ] Mueller P, Jensen K, Megonigal JP. Plants mediate soil organic matter decomposition in response to sea level rise. Global Change Biology, 2016, 22(1) : 404-414.

[6] Kwon MJ, Haraguchi A, Kang H. Long-term water regime differentiates changes in decomposition and microbial properties in tropical peat soils exposed to the short-term drought. Soil Biology \& Biochemistry, 2013, 60 : 33-44.

[ 7 ] Hou CC, Song CC, Li YC et al. Light fractions of soil organic carbon and microbial activity dynamics in marshes under different water conditions. China Environmental Science, 2012, 32(1): 113-119. [侯翠翠, 宋长春, 李英臣等. 不同水分 条件沼泽湿地土壤轻组有机碳与微生物活性动态. 中国环境科学, 2012, 32(1): 113-119.]

[ 8 ] Mou XJ, Sun ZG, Liu XT. Storage and vertical distribution characteristics of carbon and nitrogen in different Suaeda salsa marsh soils of the Yellow River Estuary. Chinese Journal of Soil Science, 2012, 43(6) : 1444-1449. [牟晓杰, 孙志高, 
刘兴土. 黄河口不同生境下翅碱蓬湿地土壤碳、氮储量与垂直分布特征. 土壤通报, 2012, 43(6): 1444-1449.]

[ 9 ] Han DL, Zhu XP, Hu Y et al. Characteristics of soil organic carbon at Bayinbuluke Swan Lake alpine wetland under different water level. Wetland Science, 2017, 15(4) : 509-515. [ 韩东亮, 朱新萍, 胡毅等. 不同水分条件下巴音布鲁克 天鹅湖高寒湿地土壤有机碳特征. 湿地科学, 2017, 15(4): 509-515.]

[10] Gao DZ, Zeng CS, Zhang WL et al. Effects of increasing flooded frequency on soil organic carbon and its active composition in the Min River estuarine wetland. Acta Scientiae Circumstantiae, 2016, 36(3) : 974-980. [高灯州, 曾从盛, 章文 龙等. 水淹频率增加对闽江口湿地土壤有机碳及其活性组分的影响. 环境科学学报, 2016, 36(3): 974-980. ]

[11] Shen YJ, Zhao QQ, Feng YQ et al. Variation characteristics of soil active organic carbon along a soil moisture gradient in a riparian zone of Taihu Lake. Chinese Journal of Ecology, 2011, 30(6): 1119-1124. [沈玉娟, 赵琦齐, 冯育青等. 太湖 湖滨带土壤活性有机碳沿水分梯度的变化特征. 生态学杂志, 2011, 30(6) : 1119-1124.]

[12] Lai JD, Tian K, Guo XL et al. Organic carbon and microbial biomass carbon in soil in Aapahia wetlands. Wetland Science, 2014, 12(1) : 49-54. [赖建东, 田昆, 郭雪莲等. 纳帕海湿地土壤有机碳和微生物量碳研究. 湿地科学, 2014,12 (1) : 49-54.]

[13] Wang DX, Gao YH, An XJ et al. Responses of greenhouse gas emissions to water table fluctuations in an alpine wetland on the Qinghai-Tibetan Plateau. Acta Prataculturae Sinica, 2016, 25(8): 27-35. [王冬雪, 高永恒, 安小娟等. 青藏高原 高寒湿地温室气体释放对水位变化的响应. 草业学报, 2016, 25(8): 27-35.]

[14] Sheng XC, Shao XX, Wu M et al. Effects of water level on organic carbon, total nitrogen and total phosphoruse in soil in reed wetlands of Hangzhou Bay. Journal of Ecology and Rural Environment, 2015, 31(5): 718-723. [ 盛宣才, 邵学新, 吴明等. 水位对杭州湾芦苇湿地土壤有机碳、氮、磷含量的影响. 生态与农村环境学报, 2015, 31(5): 718-723.]

[15] Zhang XW, Mo Y, Zhang BY et al. Effect of wetting-drying cycle and litter on dissolved organic carbon in peat soil in Zoigê Plateau. Wetland Science, 2014, 12(2) : 134-140. [张雪雯, 莫熠, 张博雅等. 干湿交替及调落物对若尔盖泥 炭土可溶性有机碳的影响. 湿地科学, 2014, 12(2) : 134-140.]

[16] Zhao GY, Guo DN, Jiang S et al. Effects of freezing and thawing on soil active organic carbon in the Xiaoxing'an Mountain wetlands. Acta Ecologica Sinica, 2017, 37 (16) : 5411-5417. [赵光影, 郭冬楠, 江姗等. 冻融作用对小兴安岭典型湿 地土壤活性有机碳的影响. 生态学报, 2017, 37(16): 5411-5417.]

[17] Guo DN, Zang SY, Zhao GY et al. Effect of wetland drainage on dissolved organic carbon biodegradation and leaching Xiaoxing'an Mountains. Acta Scientiae Circumstantiae, 2016, 36(7) : 2631-2637. [ 郭冬楠, 藏淑英, 赵光影等. 排水造 林对小兴安岭湿地土壤溶解性有机碳生物降解和淋溶的影响. 环境科学学报, 2016, 36(7) : 2631-2637.]

[18] Zhou WC, SuoLang DEJ, Cui LJ et al. Effects of drainage on soil organic carbon stock in the Zoige peatlands' eastern Qinghai-Tibetan Plateau. Acta Ecologica Sinica, 2016, 36(8): 2123-2132. [周文昌, 索郎夺尔基, 崔丽娟等. 排水对 若尔盖高原泥炭地土壤有机碳储量的影响. 生态学报, 2016, 36(8) : 2123-2132.]

[19] Zhang WM, Wu M, Shao XX et al. Changes in soil organic carbon and its active fractions during different reclamation period on the south coast of Hangzhou Bay. Journal of Soil and Water Conservation, 2014, 28(2) : 226-231. [张文敏, 吴明, 邵学新等. 杭州湾南岸不同围垦年限农田土壤有机碳及其活性组分变化. 水土保持学报, 2014, 28(2) : 226-231.]

[20] Zheng Z, Yang YF, Kong LZ et al. Changes of soil physical and chemical properties and microbial biomass in wetlands returning farmland to lake in the Caizi Lake, Anhui province. Resources and Environment in the Yangtze Basin, 2014,23 (6) : 821-826. [郑真, 杨艳芳, 孔令柱等. 退耕还湖后菜子湖湿地土壤理化性质及微生物量变化. 长江流域资源 与环境, $2014,23(6): 821-826$.

[21] Zeng ZG, Zhang XC, Liu GH et al. Structure and features of fishery resources of sub-lakes in Poyang Lake, Jiangxi, China. Resources and Environment in the Yangtze Basin, 2015, 24(6): 1021-1029. [曾泽国, 张笑辰, 刘观华等. 鄱阳湖 子湖“堑秋湖”渔业资源结构特征分析. 长江流域资源与环境, 2015, 24(6)：1021-1029.]

[22] Hu ZP, Zhang ZF, Liu YZ et al. The function and significance of the shallow-lakes in the Poyang lake wetland ecosystem. Jiangxi Hydraulic Science \& Technology, 2015, 41(5): 317-323. [胡振鹏, 张祖芳, 刘以珍等. 碟形湖在鄱阳湖湿地 生态系统的作用和意义. 江西水利科技, 2015, 41(5): 317-323.]

[23] Zhang QJ, Yu XB, Qian JX et al. Distribution characteristics of plant communities and soil organic matter and main nutrients in the Plyang Lake Nanji Wetland. Acta Ecologica Sinica, 2012, 32(12) : 3656-3669. [张全军, 于秀波, 钱建金金 等. 鄱阳湖南矶湿地优势植物群落及土壤有机质和营养元素分布特征. 生态学报, 2012, 32(12) : 3656-3669.]

[24] Jin Q, Wu Q, Zhong XZ et al. Soil organic carbon and its components under different plant communities along a water ta- 
ble gradient in the Poyang Lake wetland. Chinese Journal of Ecology, 2017, 36( 5) : 1180-1187. [ 金奇, 吴琴, 钟欣孜 等. 鄱阳湖湿地水位梯度下不同植物群落类型土壤有机碳组分特征. 生态学杂志, 2017, 36(5) : 1180-1187.]

[25] Rey A, Petsikos C, Jarvis PG et al. Effect of temperature and moisture on rates of carbon mineralization in a Mediterranean oak forest soil under controlled and field conditions. European Journal of Soil Science, 2005, 56(5) : 589-599.

[26] Janzen HH, Campbell CA, Brandt SA et al. Light-fraction organic matter in soils from long-term crop rotations. Soil Science Society of America Journal, 1992, 56(6) : 1799-1806.

[27] Hawkes CV, Waring BG, Rocca JD et al. Historical climate controls soil respiration responses to current soil moisture. Proceedings of the National Academy of Sciences of the United States of America, 2017, 114(24) : 6322.

[28] Xu XL, Zhang Q, Li YL et al. Inner-annual variation of soil water content and groundwater level in a typical islet wetland of Lake Poyang. J Lake Sci, 2014, 26(2) : 260-268. DOI: 10.18307/2014.0213. [许秀丽, 张奇, 李云良等. 鄱阳湖典 型洲滩湿地土壤含水量和地下水位年内变化特征. 湖泊科学, 2014, 26(2) : 260-268.]

[29] Davidson EA, Verchot LV, Cattanio JH et al. Effects of soil water content on soil respiration in forests and cattle pastures of eastern Amazonia. Biogeochemistry, 2000, 48(1) : 53-69.

[30] Fierer N, Craine JM, McLauchlan K et al. Litter quality and the temperature sensitivity of decomposition. Ecology, 2005, 86(2): 320-326.

[31] Knorr W, Prentice IC, House JI et al. Long-term sensitivity of soil carbon turnover to warming. Nature, 2005,433 $(7023)$ : 298-301. 